\title{
NGST: SEEING THE FIRST STARS AND GALAXIES FORM
}

\author{
H.S. STOCKMAN \\ Space Telescope Science Institute \\ 3700 San Martin Drive, Baltimore, MD 21218 \\ AND \\ JOHN MATHER \\ NASA Goddard Space Flight Center \\ Code 685, Greenbelt, MD 20771
}

\begin{abstract}
The Next Generation Space Telescope (NGST) is a key element in NASA's Origins program. The primary goals for the NGST are observing the origins of stars, galaxies, and the elements that are necessary for life. To reach those goals, the telescope must work in the near and mid-infrared at wavelengths where the Earth's atmosphere outshines the distant galaxies by up to 8 orders of magnitude. NASA, industry, US astronomers and international collaborators have completed the initial feasibility study and have begun the development of the technologies required to make the mission affordable and ready to launch by 2007 .

We describe the three missions developed in the feasibility studies: a $6 \mathrm{~m}$ monolithic telescope in a $1 \times 3$ AU elliptical orbit, and two $8 \mathrm{~m}$ deployable telescopes put into L2 halo orbits. All three telescopes are radiation cooled to temperatures of $30-60 \mathrm{~K}$. All three achieve point-source imaging sensitivities of 1 nanoJansky in a $3 \mathrm{hr}$ exposure. Such sensitivity and diffraction-limited imaging can see the birth of stellar clusters at redshifts of $z=10-20-$ even before the universe was reionized. We also illustrate $N G S T$ 's potential for studying the detailed interactions and mergers of galaxies during the peak of metal production at redshifts of $z=1-2$, an epoch when dust may mask much of the galactic light and the regions of ongoing star formation.
\end{abstract}




\section{Introduction}

Today we enjoy unprecedented facilities with which to observe the interactions between galaxies and the galactic denizens of the universe. The $H u b$ ble Space Telescope (HST) brings us breathtakingly clear views of galactic train-crashes and the strands of resulting star formation regions. The Infrared Space Observatory (ISO) peers through the blanket of dust that surrounds the most recent stellar nurseries. Sub-millimeter and millimeter arrays penetrate the nuclear cores and find dense rings of heated dust and star formation within the inner hundreds of parsecs. This symposium is a celebration of our progress and a forerunner of many years of exciting research. Within the next five years, we look forward to the operation of a dozen $8 \mathrm{~m}$-class groundbased telescopes and the Stratospheric Observatory For Infrared Astronomy (SOFIA), the construction of an international millimeter array (MMA), and the flight of the Space Infrared Telescope Facility $(S I R T F)$. Each of these will expand on our current investigations, making new detections and more quantitative measures in our nearby universe and near the apparent peak of star formation at redshifts, $z \sim 1-2$. What will follow these ambitious programs? How might astronomers and the scientific community pursue the study of the formation of and interactions between galaxies at cosmological epochs? This paper describes the potential of a new space facility, a large passively cooled telescope optimized for operation in the near infrared (NIR), the Next Generation Space Telescope (NGST).

\section{The NGST Project}

The original idea for using radiative cooling to permit the construction of large aperture infrared telescopes traces at least as far back as the early European Space Agency (ESA) designs for the Infrared Astronomy Satellite ( IRAS). It was developed further in the Edison proposal for the ESA M3 mission (Hawarden et al 1992, Thronson 1993); and it will be used for the cool-down phase of SIRTF. But the broad scientific case for such a facility was best made in the report of the HST and Beyond Committee (Dressler 1996). The charge to the committee was to consider the needs of the ultraviolet, optical, and infrared community after the end of the HST mission and the flights of other planned NASA and ESA facilities, such as SIRTF. In their recommendations, the committee identified key initiatives that would service the foreseeable scientific needs, engage the public interest, and provide a broad long range vision of ultraviolet-infrared astronomy in space. These recommendations and those of the Exploration of Neighboring Planetary Systems (ExNPS) board (Beichmann 1996) are the foundation of the NASA Office of Space Science (OSS) long range plan to search of the origins of the universe, stars, and planetary systems. The OSS initiated the 
NGST Project at Goddard Space Flight Center (GSFC), in collaboration with the Space Telescope Science Institute (STScI), to study the feasibility of developing a large ( $>4 \mathrm{~m}$ dia.) space telescope optimized for the study of the origins of galaxies such as the Milky Way galaxy. The NIR wavelength region $(\lambda=1-5 \mu \mathrm{m})$ is ideal for observing the redshifted optical light from existing stellar populations in spiral and elliptical galaxies during the peak star formation period at redshifts of $z=1-2$; with sufficient sensitivity, an NGST can detect the ultraviolet light from the very earliest formation of galaxies and stellar systems at redshifts greater than $z>10$. But the potential of a large, diffraction limited telescope in the optical and mid-IR $(\lambda=5-30 \mu \mathrm{m})$ was not ignored and the $N G S T$ Project was to consider options for extending the capabilities in these directions.

Both NASA and the HST and Beyond Committee knew that developing and operating such a telescope for a fraction of the cost of HST would be a requirement and a challenge. The goal was to break not only the cryogenic paradigm but also the constraints of existing and planned rocket shroud diameters: build, launch and operate an $8 \mathrm{~m}$ class IR telescope for less than $\$ 1 \mathrm{~B}$ (in $1996 \$$ ). The NGST Project and the NASA OSS are addressing this goal through the engagement of academia and industry and an extensive investment program in advanced technologies. In particular, the development of ultralightweight optical segments $\left(<20 \mathrm{~kg} \mathrm{~m}^{-2}\right)$, the ability to adjust them to submicron accuracy on orbit, and the availability of ultra-low noise IR detector arrays. Marshall Space Flight Center(MSFC) and the Jet Propulsion Laboratories (JPL), as well as Ames Research Center and Langley Research Center are leading many of the these technology development efforts. The goal is to bring technologies to sufficient maturity by 2003 to support an NGST construction and flight in 2007 .

In the 1996 NGST Feasibility study, three government and industry teams proposed concepts that would satisfy the scientific and fiscal objectives. These concepts are shown in Figure 1. Both the GSFC and TRW teams developed designs (shown in the upper panels) for a deployable $8 \mathrm{~m}$ telescope that would be launched to halo orbits about the L2 Lagrange point. Large multilayer sunshields would shadow the optics and instrument sections, allowing them to cool to $<60 \mathrm{~K}$ and $<30 \mathrm{~K}$ respectively. The Lockheed Martin design incorporates a $6 \mathrm{~m}$ monolithic mirror based upon the thin mirror technology developed at the University of Arizona Mirror Laboratories. A Proton or Ariane 5 launcher with a special $7 \mathrm{~m}$ dia. shroud could inject the telescope into a $1 \times 3 \mathrm{AU}$ elliptical orbit, thereby providing very low zodiacal light background for most of the $2.7 \mathrm{yr}$ orbital period. TRW and Ball Aerospace Corp. were selected in summer 1997 to consider further tradeoffs among these and other concepts. The GSFC-led team are developing detailed modeling tools to ensure that the original "Yard- 

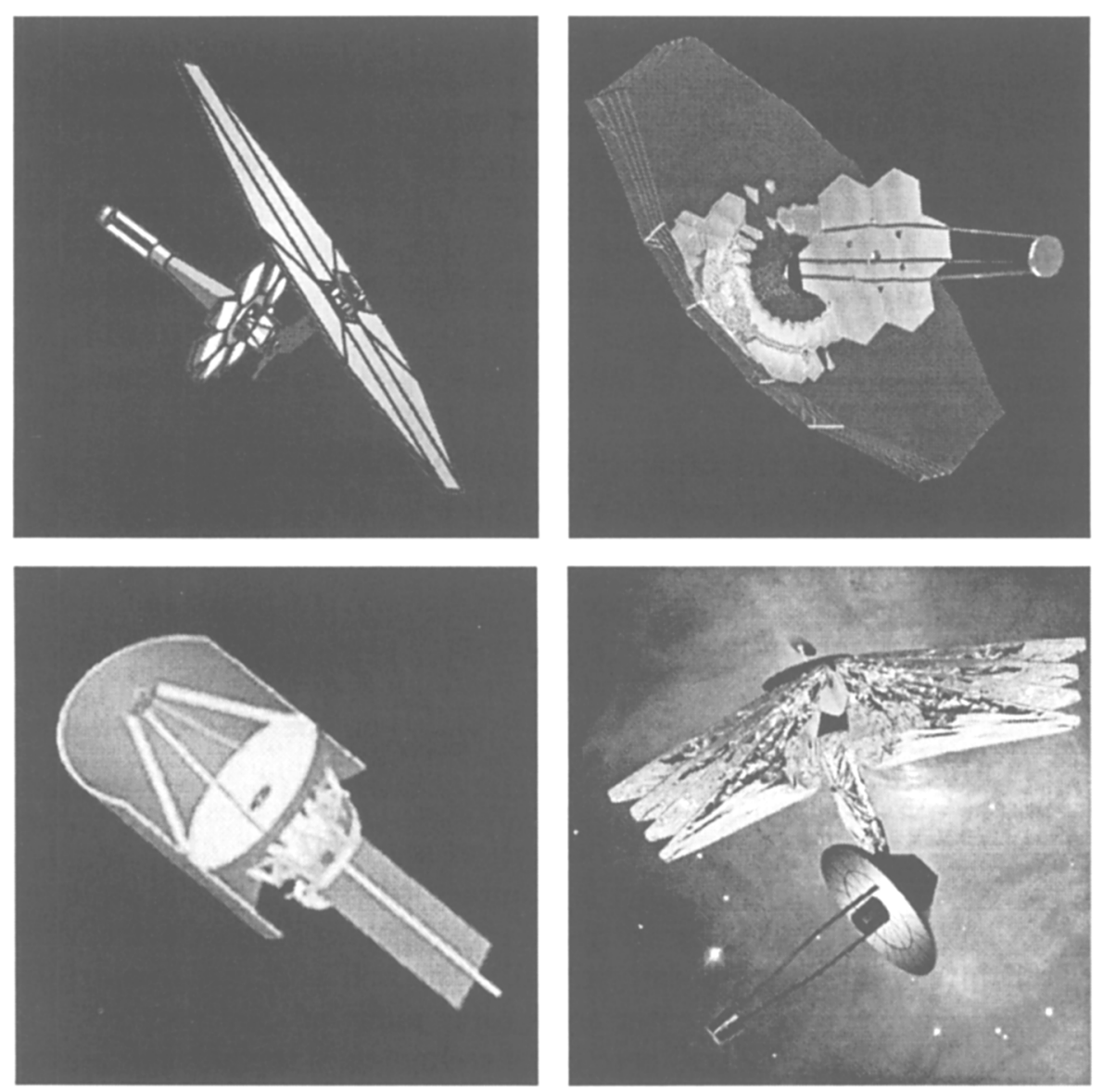

Figure 1. Four NGST Concepts. The upper left panel shows the "Yardstick" concept developed by the GSFC-led team. The upper right panel shows the TRW concept that uses a deployment technique developed by TRW for sub-mm antennae. The lower left panel displays the Lockheed Martin monolithic mirror design while the lower right panel shows a deployable concept developed by Ball Aerospace.(STScI/NASA)

stick" GSFC concept can meet the difficult thermal and pointing control requirements and to exercise different techniques for maintaining the optical alignment in space. NASA will also select five teams to develop designs for the scientific instruments. In November 1997, ESA and NASA agreed to a course that should lead to a major partnership for ESA in the NGST mission. ESA will study the feasibility of the $1 \times 3$ AU orbit, scientific instruments, and other technical areas in which ESA would lead the NGST development and operations efforts. 


\section{Studying Galaxy Formation}

The 186 IAU Symposium has provided many fascinating examples of the merging of galaxies through collisions and subsequent gravitational interactions. While small prograde portions of the galaxies may become unbound by the tidal effects during the encounters, the great majority of stars and gas eventually will coalesce into a larger unit: one with a stellar population made of the merging stellar populations and peppered with new stellar systems spawned in the merger. With star formation rates ranging between $10-100 M_{\odot} \mathrm{yr}^{-1}$ during the $10^{8} \mathrm{yr}$ after the initial encounter, the number of stars formed by mergers can be comparable to the number of stars formed in quiescent disks at $\sim 0.4 M_{\odot} \mathrm{yr}^{-1}$ for $10^{9-10} \mathrm{yr}$. In Color plate 5 (p. xxiii), we show a recent $H S T$ image of NGC 4038/4039, the Antennae, in which the star formation regions are clearly resolved into thousands of bright, revolved young globular cluster and associations. By studying the age of these young systems using their ultraviolet and optical colors, we can estimate how the star formation was triggered during the initial encounter (c.f. Whitmore and Schweizer 1995). Using NGST, we can detect these brilliant stellar nurseries at great distances and early epochs. While NGST observations of most star forming galaxies at high redshift will not achieve the resolution of the Antennae image, we expect that observations of serendipitous lensed galaxies, similar to those reported by Franx et al. (1997) will resolve the star formation regions in the transverse dimension over scales $<100$ pc. The Franx et al. (1997) images of a lensed galaxy at redshift $z=4.92$ clearly show bright star formation on scale corresponding to the clumps of globular cluster-like sources seen in Color plate 5 (p. xxiii). To demonstrate the sensitivity of $N G S T$, we show in Figure 2 the spectrum of a newly formed, large globular cluster at different redshifts compared to the sensitivity of an $8 \mathrm{~m} \mathrm{NGST}$ at L2 (1 AU) and a $6 \mathrm{~m} \mathrm{NGST}$ at $3 \mathrm{AU}$. Both telescopes would be capable of detecting such a starburst at a redshift of $z \sim 10-20\left(\Omega=1, H_{0}=50 \mathrm{~km} \mathrm{~s}^{-1}\right.$ $\left.\mathrm{Mpc}^{-1}\right)$. Even if some of the bursts are dust enshrouded like in the Antennae, the NGST would be capable of detecting interacting galaxy systems through their redshifted ultraviolet light to very great distances. Indeed, if the earliest form of star formation were through such collective star forming mechanisms, as has been suggested by Haiman and Loeb (1997), Loeb (1997), and Gnedin and Ostriker (1997), NGST would detect them.

Both far infrared and sub-millimeter observations demonstrate that many interacting systems display intense star formation tori in their remnant nuclei. Dust extinction in these systems is such that the systems are optically thick at mid-IR wavelengths (e.g. the contributions by Dave Sanders and Nick Scoville in this symposium). Except perhaps for low incli- 


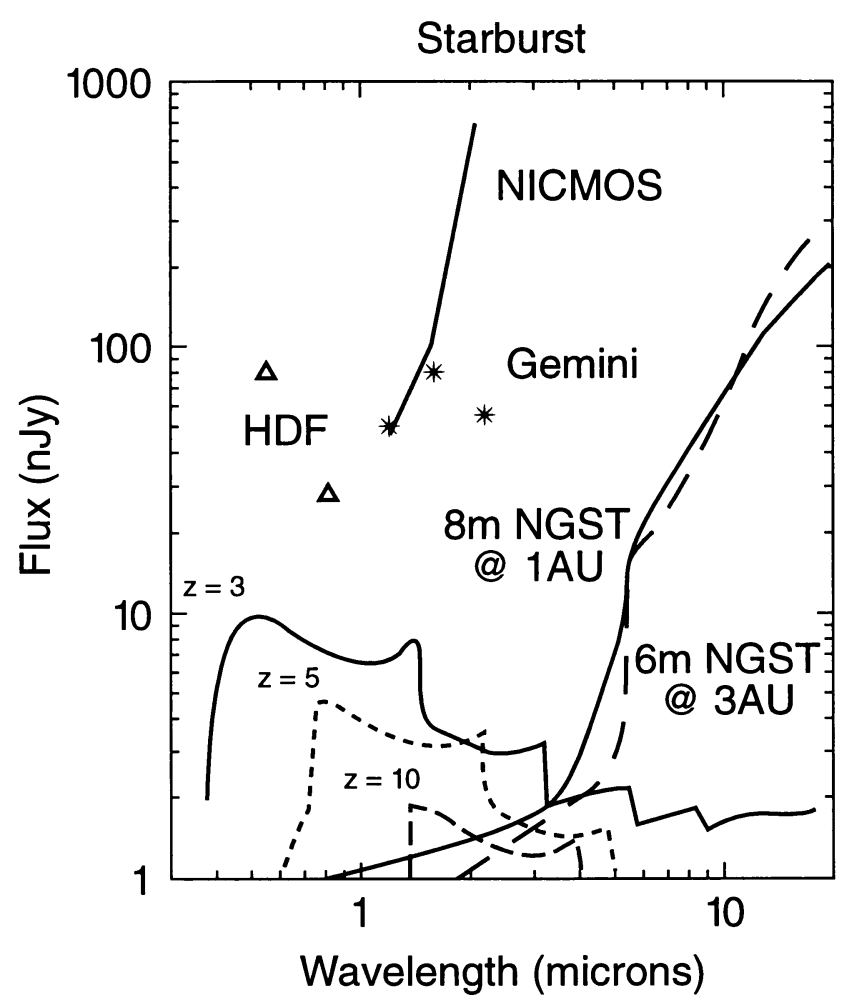

Figure 2. The spectrum from a massive starburst, $10^{6} M_{\odot}$, after 5 Myr and observed at a number of different redshifts. The spectral features are due to wind nebulae around hot stars (Leitherer and Heckman 1995). The two imaging sensitivity curves are for a

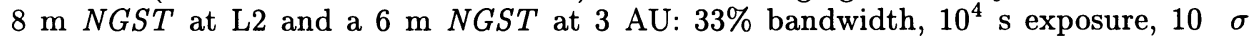
point source detections. The other curves correspond to the sensitivity of a ground-based $8 \mathrm{~m}$ telescope and adaptive optics (Gillette 1997) and $H S T /$ NICMOS. The two triangles correspond to the faintest high redshift sources ( $U$ and $B$ dropouts) detected in the Hubble Deep Field. (STScI)

nation views of these disks, neither NGST nor the IRAC camera on SIRTF will be capable of completely penetrating these regions but will be able to detect the outer skins of star formation in these tori. The recent NICMOS images of the ultraluminous IR galaxy Arp 220 easily resolves the surface of the inner torus or two separate star forming regions (Scoville et al. 1998). NGST, with 0.3 arcsec resolution at $10 \mu \mathrm{m}$, would be capable of resolving and penetrating 300 parsec dust tori at redshifts of $z \sim 1$. Because we expect that the metallicity and dust content of these systems will drop significantly at higher redshifts, NGST may be capable of detecting starforming nuclear tori to extremely large redshifts, $z>2$. Through observations such as these, coupled to deep NIR and MIR spectroscopy of 
the brighter systems and dynamic studies of quiescent galaxies - if there are any - at $z=2-3$, the $N G S T$ will be able to address the relative importance of merging on the growth and structures of galaxies such as our own. The recently selected Ad Hoc Science Working Group (ASWG) will review and develop programs such as these to direct the design trades now being studied. Other NGST scientific programs, such as the study of stellar populations in the Local Group and Virgo, will provide the fossil evidence with which to compare our understanding of the early universe to the records of steady growth and cataclysmic changes that we find around us.

We are pleased to acknowledge the strong support of $N G S T$ by Harley Thronson and Ed Weiler at NASA OSS, as well as institutional support and guidance at the GSFC and STScI. We thank Brad Whitmore and Nick Scoville for generously providing their superb $H S T$ images prior to publication. We direct interested readers to the booklet, Next Generation Space Telescope, Visiting a Time When Galaxies Were Young (Stockman 1997) and the proceedings of the 1997 Goddard Space Flight Center workshop, Science with NGST.

\section{References}

Beichmann, C.A. ed. (1996) A Road Map for the Exploration of Neighboring Planetary Systems. JPL Publ. 96-22.

Dressler, A. et al., (1996) Exploration and the Search for Origins, A Vision of Ultraviolet, Optical, and Infrared Space Astronomy. AURA, Space Telescope Science Institute.

Franx, M., Illingworth, G.D., Keson, D.D., van Dokkum, P. G., and Tran, K-V, (1997) Astrophysical Journal, 486, pp. L75-78

Gillette, F. (1997) Science with NGST, ASP Conference Proceedings, ed. E. Smith and A. Koratkar, in press

Gnedin, N.Y. and Ostriker, J.P. (1997) Astrophysical Journal, 486, pp. 581-598

Haiman, Z. and Loeb, A. (1997) Astrophysical Journal, 483, pp. 21-37

Hawarden, T. G., Cummings, R.O., Telesco, C.M., and Thronson, H.A. (1992) Space Science Reviews, 61, pp. 113-144

Leitherer, C. and Heckman, T. (1995) Astrophysical Journal Supplement, 96, pp. 9-50

Loeb, A. (1997) Science with NGST, ASP Conference Proceedings, ed. E. Smith and A. Koratkar, in press.

Mather, J.C., Smith, E.P., Seery, B.D., Bely, P.Y., Stiavelli, M., Stockman, H.S., and Burg, R. (1997) Science with NGST, ASP Conference Proceedings, ed. E. Smith and A. Koratkar, in press.

Scoville, N. Z., Evans, A.S., Dinshaw, N., Thompson, R., Rieke, M., Schneider, G., Low, F.J., Hines, D., Stobie, B., Becklin, E., and Epps, H. (1998), Astrophysical Journal, in press.

Stockman, H.S. ed. (1997) Next Generation Space Telescope, Visiting a Time When Galaxies Were Young. Space Telescope Science Institute.

Thronson, H. A. ed. (1993) Edison: an M3 Proposal to ESA. Didcot: Rutherford Appleton Labs.

Whitmore, B.C. and Schweizer, F. (1995) Astronomical Journal, 109, pp 960-980 